Published in final edited form as:

Gastroenterology. 2015 May ; 148(6): 1234-1243.e4. doi:10.1053/j.gastro.2015.02.007.

\title{
Diet and Upper Gastrointestinal Malignancies
}

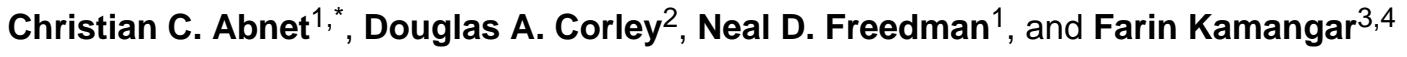 \\ ${ }^{1}$ Nutritional Epidemiology Branch, Division of Cancer Epidemiology and Genetics, National \\ Cancer Institute, Rockville, MD \\ ${ }^{2}$ Division of Research, Kaiser Permanente of Northern California, Oakland, CA \\ ${ }^{3}$ Department of Public Health Analysis, School of Community Health and Policy, Morgan State \\ University, Baltimore, MD \\ ${ }^{4}$ Digestive Oncology Research Center, Digestive Disease Research Institute, Tehran University \\ of Medical Sciences, Tehran, Iran
}

\begin{abstract}
Diet is believed to modulate cancer risk and this relationship has been widely studied in the gastrointestinal tract. Observational epidemiologic studies have provided most of the evidence for the effects of diet on cancer risk, because clinical trials to determine nutritional exposures are often impossible, impractical, or unaffordable. Although a few foods or nutrients are thought to protect against specific types of cancer, it seems clear that the strength and even direction of dietary associations (increasing or decreasing risk) is organ site- and even histology-specific, along the gastrointestinal tract. Although some hypotheses are supported by a substantial body of observational data (drinking hot maté contributes to esophageal cancer), there is not much data to support others. We discuss some highly touted hypotheses and draw interim conclusions about what is known, and what could be done to improve the level of evidence. The complex nature of diet and its associations can be productively investigated with disease-specific studies. However, public health recommendations for normal-risk individuals regarding diet and gastrointestinal cancer should probably emphasize the importance of eating for overall health, rather than eating specific foods to reduce risk for specific cancers.
\end{abstract}

\begin{abstract}
Many dietary exposures have been proposed to protect against or increase risk for gastrointestinal (GI) cancers, including diets, foods, individual nutrients, methods of preparation, and habits of consumption. Rather than a comprehensive review the vast literature published on these theories, we focus on the most critical, interesting, and popular hypotheses about the relationship between food and upper GI cancer (Table 1).
\end{abstract}

(C) 2015 Published by The AGA Institute

*Address for Correspondence: Christian Abnet, PhD, MPH, Senior Investigator, Nutritional Epidemiology Branch, Division of Cancer Epidemiology and Genetics, National Cancer Institute, 9609 Medical Center Dr Rm 6e344 MSC 9768, Bethesda MD 20892, Office: (240) 276-7213, Mobile: (240) 505-6299, abnetc@mail.nih.gov.

The authors have no competing interests to declare.

Publisher's Disclaimer: This is a PDF file of an unedited manuscript that has been accepted for publication. As a service to our customers we are providing this early version of the manuscript. The manuscript will undergo copyediting, typesetting, and review of the resulting proof before it is published in its final citable form. Please note that during the production process errors may be discovered which could affect the content, and all legal disclaimers that apply to the journal pertain. 
Epidemiologic analysis of risk factors is useful for building a case for the role of a certain exposure in cancer risk, but additional types of research are required to determine mechanisms of action. We discuss results from randomized, controlled trials when possible - this study design provides the strongest evidence for or against a specific hypothesis (data on whether supplementation with a specific food or nutrient, for a specific length of time, at a certain phase of life, alters a specific outcome). Most high-quality data on diet and upper GI malignancies come from prospective cohort studies, which assess the association between a measure of typical exposure (through questionnaire or biomarker) and future risk of disease. For uncommon diseases, such as esophageal adenocarcinoma, cohorts must be very large, to allow prospective assessment of these associations, so in some instances the best available data comes from case-control studies. There have been many publications on some exposures, reporting findings from multiple clinical trials, whereas other exposures have only been evaluated in observational studies, of varying quality.

\section{Fruits and Vegetables}

Esophageal adenocarcinoma, esophageal squamous cell carcinoma, gastric cancer, and some premalignant conditions of the upper GI tract (Barrett's esophagus) are all associated with fruit and vegetable intake, although there may be differences with location and histology. Systematic review of all studies of diet and cancer risk concluded that higher intake of fruits and vegetables probably decreases the risk of esophageal and stomach cancers. ${ }^{1}$ And some authors recommended a diet that includes at least $400 \mathrm{~g} /$ day of total fruit and vegetables. ${ }^{2} \mathrm{~A}$ more recent systematic review of 24 cohort studies of gastric cancer suggested there may be some differences between fruits and vegetables in affecting risk for gastric cancer. ${ }^{3}$ Given the limitations of data pooling, they contrasted high vs low intakes, and found a significantly reduced gastric cancer risk with fruit intake (summary relative risk (SRR), 0.90) but not vegetable intake (SRR, 0.96). However, an evaluation of dose-response found similar levels of association for fruits and vegetables: each increase of $100 \mathrm{~g}$ of intake/day of fruit was associated with a significant decrease in risk of gastric cancer (SRR, 0.95), and vegetables had a comparable, but not statistically significant association with reduced risk (SRR, $0.96) .^{3}$

Some of the effects for gastric cancer may be specific to tumor type or location, or may be restricted to persons with other risk factors. For example, in a large, prospective European study of more than 450,000 people, those with higher fruit and vegetable intakes were less likely to develop any gastric cancer (hazard ratio [HR], 0.77; $P$ for trend=.02). ${ }^{4}$ However, the associations were mainly significant for fresh fruit and diffuse-type gastric cancer (HR, 0.59; $P$ for trend=.03), for citrus and gastric cardia cancers (HR, $0.61 ; P$ for trend=.01), and for smokers and persons residing in the Northern European countries. ${ }^{4}$

Similar reductions in risk are described for esophageal carcinomas. A meta-analysis of 12 studies of esophageal adenocarcinoma associated reduced risk with higher intakes of vegetables (SRR, 0.76) and fruit (SRRs, 0.73). ${ }^{5}$ A meta-analysis of esophageal squamous cell carcinoma studies also associated decreased risk with higher-level consumption of vegetables (SRR, 0.56;) and fruits (SRR, 0.53). ${ }^{6}$ Patients with esophageal adenocarcinoma 
have been found to be less likely to consume fruits and vegetables, even after adjustment for intake of other food types (eg, meat). ${ }^{7}$

In addition to the quantity, the variety of intake may be important. In a prospective analysis of a large European cohort, within which 475 people developed gastric or esophageal cancer, increasing variety of the types of fruits and vegetables consumed, independent of total consumption, was inversely associated with the risk of esophageal squamous cell carcinoma- particularly among smokers. ${ }^{8}$

The mechanisms by which fruits and vegetables reduce cancer risk might involve their ability to prevent development of precancerous conditions. In a cohort study performed in the Netherlands, men in the highest vs the lowest quintiles of total vegetable intake had a lower risk for Barrett's esophagus (HR, 0.66;); the same was true for raw vegetable intake (HR 0.63) However, no associations were found for fruits and no significant associations were found in women. ${ }^{9}$ Somewhat similar results were found in 2 community-based casecontrol studies. In one study, persons in the highest quartile of fruit and vegetable intake were also less likely to have Barrett's esophagus (odds ratio [OR], 0.27). There was evidence for increased effects with increasing intake, even after adjusting for total energy intake. ${ }^{10} \mathrm{In}$ another study, subjects in the third tertile of fruit and vegetable intake also had a lower risk of Barrett's esophagus (OR, 0.39). ${ }^{11}$ Similar effects for vegetables were found within a veterans population, in which persons in the highest tertile of intake of dark green vegetables were less likely to have Barrett's esophagus (OR, 0.46). ${ }^{12}$ It is not clear whether these associations are directly related to chemoprotective effects of these foods, independent from other influences such as decreased acid reflux (itself a strongly associated risk factor for esophageal adenocarcinoma). An effect independent from reflux was observed in 1 study, which found a trend towards a lower risk even among persons with gastroesophageal reflux disease (OR, 0.64). ${ }^{10}$

The possible chemopreventive effects of fruits and vegetables might involve their high levels of micronutrients (including antioxidants), which can decrease DNA damage by scavenging for oxygen radicals. Foods that are high in anti-oxidants tend to be inversely associated with the risk of upper GI malignancies and telomere length. Telomere length is associated with cumulative oxidative stress from environmental factors, and telomeres are shorter in persons with gastric cancer. ${ }^{13,14}$ Fruits and vegetables might also have chemopreventive effects because they contain flavones, which inhibit cell process associated with carcinogenesis (adhesion, invasion, and migration)—possibly through their effects on focal adhesion kinase and metalloproteinases. ${ }^{15}$ Fiber may also partially mediate the associations found for fruits and vegetables.

Consumption of red or processed meat has been linked to increased risk of GI cancers. There is a convincing link with colorectal cancers and limited or suggestive evidence for links between some types of meat and other types of GI cancer. ${ }^{1,16}$ This long-standing hypothesis has been thoroughly investigated for colorectal cancer, and numerous hypotheses have been put forth to explain this apparent association. Relevant exposures include 
heterocyclic amines, $N$-nitroso compounds, and polycyclic aromatic hydrocarbons generated during specific cooking methods ${ }^{17}$, as well as the high heme-iron content of red meat. ${ }^{18}$ Processed meats have a high nitrate content, which could be particularly relevant to cancer risk.

Studies of potential mechanisms have benefited from dietary intake databases, which provide intake estimates of carcinogens from meat consumption. The CHARRED database (http://dceg.cancer.gov/tools/design/charred) provides such estimates for carcinogenic and mutagenic exposures in meat cooked under different conditions.

Studies of red meat intake must account for dietary substitution to fully model the risk associated with meat consumption. Commonly, people who reduce red meat consumption increase consumption of white meat, rather than other protein sources, so simultaneous modelling of these types of meat is important. This is particularly important given the potential for white meat to have an independent association with lower risk of cancer. ${ }^{19}$

Red meat has been frequently linked to esophageal cancer risk of both histologic types in case-control studies, whereas cohort studies have provided weaker evidence. ${ }^{20,21}$ Studies that assess meat components have suggested that heme-iron could be the most relevant factor in this potential association for esophageal adenocarcinoma. ${ }^{22}$ A similar dichotomy exists for gastric cancer; case-control studies have associated red meat with gastric cancer, whereas cohort studies have not. ${ }^{23}$

There is evidence that interactions between diet and the GI microbiome could affect cancer risk in response to different food products. Intestinal bacteria can reduce nitrate to nitrite, which can then non-enzymatically form nitrosamines, some of which are organ-specific carcinogens. Furthermore, diet strongly and quickly changes the microbial populations in the colon. ${ }^{24}$ The production of trimethylamine- $N$-oxide by intestinal microbes from choline in red meat has been linked to atherosclerosis ${ }^{25,26}$. However, further studies are needed to establish a link between specific intestinal microbes or their products and cancer risk.

\section{Hot beverages}

The relationship between hot drinks, including black and green tea, coffee, and maté (an infusion of the herb Ilex Paraguarensis), and GI cancers have been studied extensively. Epidemiologic studies have been conducted since at least the 1960s and clinical studies date even further back. For example, in 1939, WL Watson reviewed clinical records from 771 esophageal cancer patients and concluded that: "thermal irritation is probably the most constant factor predisposing to the cancer of the esophagus". ${ }^{27}$ Despite this long history of clinical and epidemiologic studies, the only established association thus far seems to be that of maté drinking and esophageal squamous cell carcinoma. ${ }^{28,29}$ Other widely discussed associations, such as that between hot tea drinking and esophageal squamous cell carcinoma, remain uncertain. ${ }^{29}$

Hot tea and coffee drinking were proposed to be factors that could cause esophageal cancer, mostly squamous type, based on the hypothesis that recurrent thermal injury could cause cancer. Support for this hypothesis came from studies by De Jong and colleagues, which 
showed that drinking hot beverages could substantially increase the intra-esophageal temperature, and this increase was a function of the initial drinking temperature and more importantly, the size of the sip. ${ }^{30}$ Drinking $65^{\circ} \mathrm{C}$ coffee increased the intra-esophageal temperature by $6-12{ }^{\circ} \mathrm{C}$, depending on the sip size.

However, epidemiologic studies have not consistently shown associations between hot tea or coffee consumption and risk of esophageal cancer. ${ }^{29}$ There is almost no evidence that coffee increases the risk of esophageal cancer. In fact, most new studies have suggested that coffee may protect against esophageal cancer. ${ }^{31,32}$ Whereas several epidemiologic studies have shown an increased risk of esophageal cancer with drinking hot tea, others have shown no association or even a reduced risk. ${ }^{29}$ Studies in northern parts of Iran - where esophageal squamous cell carcinoma and hot tea drinking are common - have shown a relatively strong positive association between the two. ${ }^{33}$ Similarly, studies performed in Kashmir, India reported a positive association between hot tea drinking and risk of esophageal squamous cell carcinoma. ${ }^{34}$ However, many other studies, including several large-scale studies from Europe and China, have shown no or even protective associations. ${ }^{32,35,36}$ Therefore, it appears that if there is any risk, it is only for those who drink large amounts of burning-hot tea.

There are several reasons that extensive research over the past few decades has not produced consistent findings. One reason involves the heterogeneity in the chemical constituents of tea, coffee, and maté. Although some studies have reported mutagenic effects of tea, coffee, and unprocessed maté herb extracts ${ }^{37,38}$, others have reported that they may prevent cancer. ${ }^{39,} 40$ Maté, in particular, can have substantial amounts of mutagenic polycyclic aromatic hydrocarbons. ${ }^{41}$ Conversely, many studies have reported that some green tea polyphenols, most notably i(-)-epigallocatechin-3-gallate, have anti-neoplastic properties, suppressing cell proliferation, inhibiting angiogenesis, and increasing apoptosis. ${ }^{42}$ Tea polyphenols have also been reported to act on the immune system, affecting T-cell functions. ${ }^{43}$ However, most of these studies were performed in cell lines or using animal models. Human epidemiologic studies have not consistently associated decreased risk of esophageal cancer with green intake; a recent meta-analysis found essentially no effect on risk. $^{44}$

There have been some troubling issues with study designs and methods (retrospective design, questionnaires with subjective questions and incomplete responses, and lack of information on histologic type of esophageal cancer), incomplete analyses, and data reporting. ${ }^{29}$ For example, few studies measured the actual temperature of tea, coffee, or maté that were consumed by participants, or obtained data on amount or frequency of drinking per day, total duration of drinking, or sip size (or an indicator of this). New, welldesigned studies are underway with consideration of these factors. ${ }^{45}$

Other studies have investigated associations of tea, coffee, or maté with other types of GI cancers, such as gastric and colorectal cancer. ${ }^{46,}{ }^{47}$ However, those associations are not well established. One of the more interesting and perhaps most-established hypotheses is a role for coffee in preventing liver cancer. 


\section{Micronutrients, Anti-oxidants, and Multi-vitamins}

Considering the difficulty of increasing fruit and vegetable intake, at a population level, studies have tried to identify which components of fruits and vegetables are beneficial. ${ }^{48}$ Of the many constituents in fruits and vegetables, antioxidant micronutrients and fiber have likely received the most attention. Many carcinogens are thought to damage DNA by generation of reactive oxygen species. Anti-oxidants such as vitamin $\mathrm{C}$ and $\mathrm{E}, \beta$-carotene, selenium, and others, may prevent this oxidative damage and help maintain a normal redox cellular environment. ${ }^{49}$ Supplementation or fortification of the diet with micronutrients, either individually or in combination, could ensure that people have sufficient intakes of micronutrients and help prevent cancer.

Half of adults in the United States (US) ${ }^{50}$ regularly use micronutrient supplements. Although there is established evidence for the positive effects of micronutrient supplements in subgroups, such as pregnant women ${ }^{51}$ and patients with GI conditions such as pernicious anemia, ${ }^{52}$ there is surprisingly little evidence that the supplements prevent cancers of the GI tract or other tissues. ${ }^{53,54}$ The effects of micronutrient anti-oxidants have been examined in laboratory, observational, and randomized controlled trials. ${ }^{54-57}$ There is evidence from observational studies for an inverse association between serum levels of $\beta$-carotene, retinol, selenium, vitamin E, and other micronutrients and risk of upper GI cancer. ${ }^{58-61}$ However, foods typically contain multiple micronutrients, so findings for one micronutrient could be confounded by another, or by other features of diet and lifestyle. Furthermore, findings in populations, such as central China, that have numerous nutrient deficiencies might not apply to well-nourished populations in other parts of the world.

Prospective, controlled trials are needed to study the effects of micronutrients. However, due to considerable design challenge, few such trials have been conducted for less common cancers. Key considerations ${ }^{62}$ include whether studies should examine single micronutrients, such as vitamin D or a multi-vitamin. How should the vitamins be formulated and what concentrations should be given? Should studies be conducted among nutritionally deficient populations, whom supplementation is most likely to show a benefit, or among well-nourished populations, which already consume the bulk of micronutrient supplements worldwide?

Perhaps the strongest data in support of micronutrient supplementation and upper GI malignancies comes from a trial of 29,584 participants in Linxian, China, where many people had nutritionally deficencies. ${ }^{63}$ Participants who received factor D (15 mg $\beta$ carotene, $50 \mu \mathrm{g}$ selenium, and $30 \mathrm{mg} \alpha$-tocopherol) daily for 5.25 years had a lower risk of gastric cancer at the end of the trial and after an additional 10 -year post-supplementation follow-up period (HR, 0.89). However, whether such findings apply to well-nourished populations is unclear.

Another important issue is the complexity and nearly always prohibitive cost of powering trials large enough to examine effects on particular cancer types. For example, the Physician Health Study II included 14,641 men and observed a beneficial effect of 13.8 ys of multivitamin supplementation on overall cancer incidence (HR, 0.92). However, even with this 
relatively large size, the study had only modest power to examine individual cancers. Importantly, results from the few other trials that examined multi-vitamins did not find evidence for an effect, even among the nutritionally deficient Linxian population. ${ }^{64}$

Alternatively, trials focused on intermediate endpoints can be smaller and cost much less. However, findings for intermediate endpoints may not necessarily apply to cancer. ${ }^{65}$ Data from the Pioglitazone or Vitamin E for NASH Study (PIVENS) found that vitamin E supplementation improved histologic features of patients with non-alcoholic steatohepatitis over 96 weeks. ${ }^{66}$ However, the effects of vitamin E supplementation on long termoutcomes, including liver cancer, are not clear. ${ }^{67}$ Data from observational studies, including prospective cohort studies, might also be evaluated. However, results from these types of studies can differ from those of randomized, controlled trials. Perhaps the most striking example of this occurred in studies of the effects of $\beta$-carotene supplements on lung cancer. Observational studies found that $\beta$-carotene supplementation might protect smokers from lung cancer, ${ }^{68}$ whereas later trials actually found that $\beta$-carotene increased cancer risk. ${ }^{69,70}$ Studies of selenium and vitamin $\mathrm{E}$ and prostate cancer made analogous findings. The Selenium and Vitamin E Cancer Prevention Trial (SELECT) reported that randomization to these nutrients was associated with non-significantly higher prostate cancer risk, ${ }^{71}$ in contrast to previous findings.

So, despite interesting hypotheses, few large-scale randomized trials have examined the effects of micronutrient supplementation on chronic disease, and even fewer focused on upper GI malignancies. As reflected in current guidelines ${ }^{72}$ and consensus reports, ${ }^{73}$ data do not support the widespread use of micronutrient supplements by much of the adult US population, and there is even some evidence that multi-vitamin supplementation might be harmful.

\section{Vitamin D}

Vitamin $\mathrm{D}$ is a hormone that in most people is primarily formed by the skin during exposure to ultraviolet light. Diet, including fortified milk in the US, also provides vitamin D. Vitamin D was discovered because of its activities in maintaining bone health and preventing rickets, but in recent years there has been an explosion of hypotheses about its effects. Reduced levels of vitamin D have been proposed to increase risk for cancers of the colorectum, esophagus, pancreas, and other organs. Recently, the US Institute of Medicine comprehensively reviewed our knowledge about vitamin D and concluded that bone health is the only proven benefit of sufficient vitamin D intake. ${ }^{79}$ Nevertheless, the epidemiological evidence evaluating the association between vitamin and cancer in humans is growing rapidly. Prospective studies of serum levels of $25(\mathrm{OH})$ vitamin D have mostly associated lower concentrations with increased risk for colorectal cancer. ${ }^{80}$

Vitamin D binds to the vitamin D receptor-a ligand-activated transcription factor that regulates a number of biochemical processes and can reduce cell proliferation. ${ }^{81}$ Vitamin D is also thought to have substantial effects on the immune system, including stimulating antigen-presenting and T-regulatory cells, increasing expression of anti-microbial peptides, and regulating T-helper 1 and 2 cells. $^{82}$ Serum 25(OH)D is the most widely used biomarker 
of vitamin D exposure. However, researchers have recently started to measure and assess the role of the vitamin D binding protein, which may have an independent or interactive effects on cancer risk. ${ }^{83,84}$

Cross sectional case-control studies have examined the role of vitamin D in cancer incidence, but the utility of these studies is suspect. Notable differences in findings from cross-sectional and prospective studies of vitamin D and breast cancer suggest that lower vitamin D concentrations could be an effect, rather than a cause, of cancer development. ${ }^{85}$ The discovery of at least 4 common polymorphisms that are strongly associated with circulating vitamin D concentrations ${ }^{86,87}$ has allowed for instrumental variable studies to evaluate vitamin D's effect on cancer risk ${ }^{88}$.

Studies of the association between vitamin D levels and cancers of the upper GI tract have produced mixed results; some studies have associated high, or higher, serum levels of vitamin D with increased risk of esophageal squamous cell carcinoma ${ }^{89}$, its precursor lesion esophageal squamous dysplasia ${ }^{90}$, and pancreatic cancer ${ }^{91}$. However, no effects were observed on risk for gastric cancer. ${ }^{89}$ A number of less-common cancers were studied in the Vitamin D Pooling Project (VDPP), ${ }^{92}$ including upper GI cancers ${ }^{93}$ and cancers of the pancreas. ${ }^{94}$ The VDPP showed no evidence that higher concentrations of vitamin D protect against either of these cancers. In fact, there was some evidence that vitamin D was associated with increased risk of upper GI cancer in some subgroups. Findings from studies of pancreatic cancer have produced mixed results, reporting that high levels of vitamin D increase risk ${ }^{91}$, have no effect on risk ${ }^{95}$, or reduce risk. ${ }^{96}$ Given the complex and contradictory evidence from observational studies, it seems that large, well-powered clinical trials may be required to resolve the controversy regarding vitamin D and risk of these cancers.

\section{Pickled vegetables}

Asian pickled vegetables were suspected to have a role in the etiology of esophageal squamous cell carcinoma, due to their high intake in areas of China where this cancer is prevalent. Analyses of cancer registries from the 1960s revealed an extremely high incidence of esophageal cancer in Taihang Mountain regions of China. ${ }^{97}$ Well-established causes of esophageal squamous cell carcinoma, such as tobacco and alcohol consumption, could not account for this high incidence ${ }^{98}$, so other factors, ${ }^{99}$ particularly intake of pickled vegetables, were investigated.

In these areas of China, pickled vegetables were an integral part of the diet of many families and were eaten 9-12 months of the year. ${ }^{98}$ In the traditional method of preparation, moist vegetables are kept tightly packed in a jar for a few months, allowing fermentation and growth of fungi and yeast, ${ }^{99,} 100$ which can generate potentially carcinogenic mycotoxins and $N$-nitroso compounds. ${ }^{99,101,102}$ Pickled vegetables have shown mutagenic and carcinogenic effects in some animal and in vitro studies. ${ }^{99,103,104}$

Nonetheless, a 1993 report from the International Agency for Research on Cancer (IARC) Working Group ${ }^{100}$ concluded that there was limited evidence from humans and inadequate evidence from animals to support the carcinogenicity of pickled vegetables. Whereas some 
human observational studies, including case-control and cohort studies, conducted before 1993 reported an increased risk of esophageal squamous cell carcinoma, others had not.

Two meta-analyses summarized the results of human studies of pickled vegetables, one in relation to esophageal cancer ${ }^{105}$ and the other in relation to gastric cancer. ${ }^{106}$ The first metaanalysis ${ }^{105}$, which included 34 studies, showed a statistically significant 2 -fold increase in risk of esophageal squamous cell carcinoma (OR, 2.08). Several sensitivity analyses confirmed the increased risk, and most subgroup analyses showed a statistically significant association increased risk. However, most data were obtained from retrospective studies, so well-designed prospective studies are warranted. The second meta-analysis ${ }^{106}$ included 60 studies (49 case- control and 11 cohort). Thirty of the case-control studies found that pickled vegetables significantly increased risk for gastric cancer, and 1 found that it significantly reduced risk. Among prospective studies, 2 found that the pickled vegetables significantly increased risk of gastric cancer, and none found a significant decrease in risk. OR values were 1.52 for an overall association, 1.56 from the case-control studies, and 1.32 from the cohort studies.

Since 1993, there has been increasing overall evidence that pickled vegetables might cause esophageal and gastric cancers so it seems reasonable that people be advised to avoid these products or use methods of preparation that avoid production of carcinogens.

\section{Salt}

The World Cancer Research Fund/American institute for Cancer Research (WCRF/AICR) said that: "Salt, and also salt-preserved foods, are probably causes of [gastric cancer]". 107 Likewise, a World Health Organization/Food and Agriculture Organization (FAO) expert panel concluded that salt is a probable cause of gastric cancer. ${ }^{108}$

Salt intake was initially associated with gastric cancer in ecological studies that compared areas at high and low risk for gastric cancer. A 1965 study by Joossens et al, correlated mortality from stroke and gastric cancer across geographic areas and over time, and proposed that salt might be a shared risk factor for both diseases. ${ }^{109}$ A 1996 study 110 showed a more direct link between salt and gastric cancer. In this study, urine samples collected over 24-hr periods, from 39 populations in 24 countries, correlated gastric cancer mortality rates with salt excretion in urine ( $\mathrm{R}$ values of .70 in men and 0.74 in women; both $p<0.001)$.

It is difficult to measure salt intake in human studies. However, people can report their preference for salty vs non-salty foods. So, some epidemiologic studies ask the participants about their preference for salty food, ${ }^{111}$ in addition to, or in lieu of, measuring total salt intake. Overall, findings from epidemiologic studies support the association between higher salt intake and increased risk of gastric cancer. ${ }^{112} \mathrm{~A}$ meta-analysis of 10 cohort studies, including nearly 269,000 individuals and 1474 diagnosed cases, showed an incrementally increasing risk of gastric cancer with higher salt intake. ${ }^{13}$ Compared to people who consumed low levels of salt, those with moderate and high salt intake were at increased risk for gastric cancer; RR values were 1.41 and 1.68 , respectively. ${ }^{113}$ 
There are several mechanisms by which salt might contribute to gastric carcinogenesis. Ingestion of salt causes gastritis in rats, and salt increases the effects of gastric carcinogens such as $N$-methyl- $N$-nitro- $N$-nitrosoguanidine. ${ }^{114,115}$ High salt concentration in the stomach can disrupt the mucosal barrier and lead to inflammation and atrophy. Such mucosal damage has been to shown to increase Helicobacter pylori colonization in mice ${ }^{116}$ and perhaps humans. ${ }^{117}$ Mongolian gerbils with cagA-positive $H$ pylori infections placed on a high-salt diet developed more severe gastric inflammation than those on a regulate diet, and also had higher gastric $\mathrm{pH}$ and increased loss of parietal cells. ${ }^{118}$ Furthermore, animals fed a high-salt diet had increased transcription of cagA, compared to those on a regular diet. These findings indicate that a salt-rich diet can potentiate the carcinogenic effects of $c a g A$-positive strains of $H$ pylori. ${ }^{118}$

\section{Coffee}

Despite its widespread popularity, coffee drinking is not typically considered among the pantheon of healthy behaviors. Many people even consider coffee drinking to be their guilty pleasure. The origins of such feelings likely stem from the presence of the drug caffeine in coffee; side effects such as insomnia, anxiety, and heartburn; withdrawal symptoms including headaches and fatigue; and concerns about long-term health effects. However, there is much evidence that coffee drinking has beneficial effects for patients with chronic diseases, ${ }^{119-121}$ including GI malignancies.

There is most evidence for the effects of coffee on liver cancer. ${ }^{122}$ However, some studies have found inverse associations with colorectal ${ }^{123,124}$ and esophageal cancers ${ }^{125}, 126$, but generally not gastric cancer. ${ }^{127}$ The most recent meta-analysis of liver cancer studies found that the RR for low-level consumption of coffee was 0.72 , and for high-level consumption was 0.44 , compared with not drinking coffee. ${ }^{122}$ Consistent findings have been reported from case-control and prospective cohort studies, in populations worldwide, including regions with distinct spectrums of liver cancer risk factors (China, Japan, and Europe).

In addition to liver cancer, inverse associations have been observed between coffee intake and a range of liver disease markers and outcomes in cross-sectional studies, including fibrosis, ${ }^{128}$ levels of liver enzymes ${ }^{129-131}$, and fatty liver ${ }^{132}$. In patients with hepatitis C virus infection, coffee was reported to affect response to treatment with peg-interferon and ribavirin ${ }^{133}$ and progression of advanced-stage disease. ${ }^{134}$ Coffee drinking has also been associated with incidence and mortality from chronic liver disease and cirrhosis in several cohort studies. ${ }^{135-140}$

Such findings are intriguing. ${ }^{141-144}$ However, a number of important questions must be answered before the association can be considered to be definitive. First, little is known about the mechanisms ${ }^{145}$ by which coffee protects the liver. Coffee contains more than 1000 compounds, and we don't know which ones are important. Few studies have evaluated differences in effects of caffeinated vs decaffeinated coffee ${ }^{130}$, let alone examined the possible effects of coffee roasting or brewing methods ${ }^{139}$ on the compounds in coffee and associations with disease. 
Coffee might affect the immune system; coffee drinking was inversely associated with markers of systemic inflammation in several studies. ${ }^{146-149}$ Coffee, and compounds within, have also been shown to affect important immune signaling pathways, such as the JNK to STAT and transforming growth factor- $\beta$ signaling. ${ }^{150-152}$ Coffee might also affect liver cancer development by modulating energy metabolism, because coffee drinking has been inversely associated with diabetes in a large number of epidemiology studies. ${ }^{153}$ Coffee is also one of the leading sources of micronutrients in the US diet, although, as described elsewhere, the effects of micronutrients on liver cancer are unclear.

Second, nearly all studies have been observational in design, making it difficult to prove that coffee itself affects liver disease development. Confounding, reverse causality, and bias could account for observed associations, so this hypothesis could be strengthened by randomized trials. Yet, it will be challenging to determine the appropriate setting and design for such studies. For example, most people have a firm opinion as to whether they like drinking coffee or not, so it would difficult to assign people to groups that do or do not drink coffee- particularly for a long-term study. Nonetheless, in spite of increasing numbers of observational studies finding inverse associations between coffee drinking and liver cancer, further research is needed to demonstrate that coffee itself affects liver cancer development, and to identify its potential mechanisms.

\section{Alcohol}

Alcoholic beverages are widely consumed and serve many important roles in economics, society, and social rituals. However, alcohol also poses a major conundrum to public health. Abusive consumption of alcoholic beverages leads to public health problems that include direct effects on the consumer (liver disease, motor vehicle accidents, increased cancer risk) and indirect effects on those around them (social and familial instability, motor vehicle accidents, etc). Yet modest consumption of alcoholic beverages has been consistently linked to lower over mortality ${ }^{154}$, mainly via reduced risk of heart disease. ${ }^{155}$

The association between alcohol consumption and cancer risk is complex. Alcoholic beverages are classified as class 1 carcinogens by the IARC; this was previously construed as the beverages in total, not as ethanol. But the most recent update has expanded the group 1 classification from alcohol consumption to the ethanol in alcoholic beverages and the acetaldehyde associated with consumption of alcoholic beverages. ${ }^{156}$ It seems likely that alcoholic beverages have multiple carcinogenic mechanisms, given the wide range of associations with cancer. For example, there seems to be a monotonic, possibly linear, association between higher consumption of alcohol and risk of breast cancer, such that even moderate intake could increase risk. ${ }^{157}$ On possible mechanism is that alcohol can influence sex hormone metabolism. ${ }^{158}$ In contrast, greater consumption of alcohol has been associated with reduced risk of kidney cancer, even at high levels of alcohol intake. ${ }^{159}$ The diuretic effect of alcohol may keep urine dilute, thereby reducing exposure of kidney tissues to urinary mutagens.

In contrast to breast and kidney cancers, alcoholic beverages have a complex set of associations with GI cancers (Figure 1). Risk of esophageal squamous cell carcinoma is 
closely tied to heavy alcohol consumption in Western countries, with a populationattributable fraction of about $72 \% .{ }^{160}$ But in many studies this association does not appear to be monotonic. For example, in the NIH-AARP ${ }^{161}$ and Million Women cohorts ${ }^{162}$, abstaining subjects did not have the lowest risk for upper GI squamous cell carcinomas, and subjects who consumed larger amounts of alcohol had higher risks. This association has been reported in other studies, but some studies have not classified exposure in metrics that lend themselves to testing for this effect, collapsing modest and non-consuming subjects into a single referent category. It could be possible that the non-drinker category includes subjects who were formerly heavy drinkers, which alters the estimate of risk in this group. Or, modest alcohol consumption could be confounded by the fact that many people in this category have otherwise healthy diets and lifestyles. Or the true association could be nonlinear, with protection afforded by an unknown mechanism.

Many people of East Asian ancestry have polymorphisms that affect metabolism of ethanol, commonly known as the flushing response. ${ }^{163}$ Individuals with a single copy of a specific polymorphism in $A L D H 2$ develop symptoms after consumption of ethanol, so they eliminate it from their diet. Subjects that continue to drink alcoholic beverages are at extraordinarily high risk of developing esophageal squamous cell carcinoma, with estimated ORs $>60 .{ }^{163}$ Subjects that carry 2 alleles of this polymorphism generally cannot tolerate any ethanol consumption and are therefore protected from cancer. This difference may reflect differences in the effects of modest alcohol consumption in the general population. ${ }^{164}$

Alcohol seems to have little or no role in the etiology of esophageal adenocarcinoma. An analysis from the Barrett's and Esophageal Adenocarcinoma Consortium (BEACON) found no association between total alcoholic beverage consumption, even high doses ( 7 drinks/ day) and esophageal adenocarcinoma. ${ }^{161}$

In most studies, adenocarcinomas of the stomach appear not to be associated with alcoholic beverage consumption, but there is some evidence for an adverse effect in subjects with genetic polymorphisms that alter metabolism of ethanol, similar to the effects of $A L D H 2$ variants on esophageal cancer risk, although the current level of evidence is modest. ${ }^{165,} 166$ Heavier consumption of alcohol has been causally linked to cancers of colorectum, but with a more modest magnitude than for other types of GI cancers, such as esophageal squamous cell carcinoma. There is some evidence that the increase in risk could be linked to the depletion of folate with heavy alcohol consumption.

\section{Dietary Patterns}

Separate from individual foods or food groups, dietary patterns are also associated with cancer risk and may offer a more powerful alternative to food-by-food studies. One approach for evaluating such patterns is the use of principal components analyses, which can identify independent associations for groups of dietary elements that are associated with each other, but not associated with other dietary elements. This method assesses shared dietary patterns that occur in the general population. A population-based case-control study in performed in Connecticut, New Jersey, and western Washington State used this type of analysis to evaluate subtypes of esophageal and gastric cancers, patterns of food intake, and 
other risk factors (eg, body mass index). ${ }^{167}$ The study found that dietary patterns with higher intakes of fruits and vegetables, as scored by the principal components analysis, were associated with decreased risks for esophageal squamous cell carcinoma (OR, 0.36), esophageal adenocarcinoma (OR, 0.43), and gastric adenocarcinoma (OR, 0.63). The dietary pattern higher in meat and nitrates was strongly associated with an increased risk of esophageal adenocarcinoma (OR, 5.61), and somewhat more weakly with increased risks of esophageal squamous cell carcinoma and non-cardia gastric adenocarcinomas (OR, 2.40 for fourth vs first quartiles).

A study that used these methods to study Barrett's esophagus found similar results. In that study, a more health-conscious dietary pattern, with higher intakes of fruits, vegetables, and non-fried fish, was associated with a decreased risk of Barrett's esophagus (OR, 0.35). ${ }^{168} \mathrm{In}$ contrast, subjects with Western-style dietary patterns, including fast-foods and meat, were more than 2-fold more likely to have Barrett's esophagus (OR, 2.30), although without a strong dose effect and with non-significant results for the fourth quartile of intake. ${ }^{168}$

Alternatively, dietary pattern analysis can use a priori dietary patterns that are thought to optimize health outcomes (often defined using knowledge of diet and heart disease), such as the healthy eating index ${ }^{169}$ or the Mediterranean diet. ${ }^{170}$ Several recent studies have assessed the association between such patterns and risk of GI cancer. These generally find that pre-specified healthy diets are associated with lower risk of GI cancer.

\section{Implications and Future Directions}

Diet seems to have an important role in risk for GI cancer, but it is difficult to draw firm conclusions, except for some strongly adverse exposures, such as some forms of meat and colorectal cancer risk, or the consumption of extremely hot beverages and esophageal cancer risk. Future observational studies must be primarily prospective, due to the difficulty of excluding bias when examining evidence derived from cross-sectional case-control studies. Trials can be helpful, but are not always possible, and negative results do not always quell the perception that modulating intake of certain foods or nutrients will lower risk for GI malignancies.

The potentially protective effect of coffee on liver cancer seems ready to test in a randomized trial, if a suitable population can be identified. Several mechanisms of action are likely to be involved, including effects on the immune system. Future studies of coffee and liver cancer may be more informative if mechanistic analyses are involved. The causes of upper GI cancers are relatively under-studied, compared to cancer types that are more common in high-income countries. Finally, analyses of dietary patterns are intriguing and might be used to educate the public to eat for overall health, rather than focusing on individual foods or nutrients and their effects on specific cancers, at individual organ sites.

\section{Acknowledgments}

This work was supported by the Intramural Research Program of the National Institutes of Health, National Cancer Institute. 


\section{References}

1. World Cancer Research Fund / American Institute for Cancer Research. Food, Nutrition, Physical Activity, and the Prevention of Cancer: a Global Perspective. Washington, D.C.: AICR; 2007.

2. Key TJ, Schatzkin A, Willett WC, et al. Diet, nutrition and the prevention of cancer. Public Health Nutr. 2004; 7:187-200. [PubMed: 14972060]

3. Wang Q, Chen Y, Wang X, et al. Consumption of fruit, but not vegetables, may reduce risk of gastric cancer: results from a meta-analysis of cohort studies. Eur J Cancer. 2014; 50:1498-1509. [PubMed: 24613128]

4. Gonzalez CA, Lujan-Barroso L, Bueno-de-Mesquita HB, et al. Fruit and vegetable intake and the risk of gastric adenocarcinoma: a reanalysis of the European Prospective Investigation into Cancer and Nutrition (EPIC-EURGAST) study after a longer follow-up. Int J Cancer. 2012; 131:29102919. [PubMed: 22473701]

5. Li B, Jiang G, Zhang G, et al. Intake of vegetables and fruit and risk of esophageal adenocarcinoma: a meta-analysis of observational studies. Eur J Nutr. 2014; 53:1511-1521. [PubMed: 24448974]

6. Liu J, Wang J, Leng Y, et al. Intake of fruit and vegetables and risk of esophageal squamous cell carcinoma: a meta-analysis of observational studies. Int J Cancer. 2013; 133:473-485. [PubMed: 23319052]

7. Navarro Silvera SA, Mayne ST, Risch H, et al. Food group intake and risk of subtypes of esophageal and gastric cancer. Int J Cancer. 2008; 123:852-860. [PubMed: 18537156]

8. Jeurnink SM, Buchner FL, Bueno-de-Mesquita HB, et al. Variety in vegetable and fruit consumption and the risk of gastric and esophageal cancer in the European Prospective Investigation into Cancer and Nutrition. Int J Cancer. 2012; 131:E963-E973. [PubMed: 22392502]

9. Keszei AP, Schouten LJ, Driessen AL, et al. Vegetable, fruit and nitrate intake in relation to the risk of Barrett's oesophagus in a large Dutch cohort. Br J Nutr. 2014; 111:1452-1462. [PubMed: 24308274]

10. Kubo A, Levin TR, Block G, et al. Dietary antioxidants, fruits, and vegetables and the risk of Barrett's esophagus. Am J Gastroenterol. 2008; 103:1614-1623. [PubMed: 18494834]

11. Thompson OM, Beresford SA, Kirk EA, et al. Vegetable and fruit intakes and risk of Barrett's esophagus in men and women. Am J Clin Nutr. 2009; 89:890-896. [PubMed: 19144726]

12. Jiao L, Kramer JR, Rugge M, et al. Dietary intake of vegetables, folate, and antioxidants and the risk of Barrett's esophagus. Cancer Causes Control. 2013; 24:1005-1014. [PubMed: 23420329]

13. Hou L, Savage SA, Blaser MJ, et al. Telomere length in peripheral leukocyte DNA and gastric cancer risk. Cancer Epidemiol Biomarkers Prev. 2009; 18:3103-3109. [PubMed: 19861514]

14. Chung MY, Lim TG, Lee KW. Molecular mechanisms of chemopreventive phytochemicals against gastroenterological cancer development. World J Gastroenterol. 2013; 19:984-993. [PubMed: 23467658]

15. Lin SH, Shih YW. Antitumor effects of the flavone chalcone: inhibition of invasion and migration through the FAK/JNK signaling pathway in human gastric adenocarcinoma AGS cells. Mol Cell Biochem. 2014; 391:47-58. [PubMed: 24510324]

16. Abid Z, Cross AJ, Sinha R. Meat, dairy, and cancer. Am J Clin Nutr. 2014; 100:386S-393S. [PubMed: 24847855]

17. Kim E, Coelho D, Blachier F. Review of the association between meat consumption and risk of colorectal cancer. Nutr Res. 2013; 33:983-994. [PubMed: 24267037]

18. Cross AJ, Ferrucci LM, Risch A, et al. A large prospective study of meat consumption and colorectal cancer risk: an investigation of potential mechanisms underlying this association. Cancer Res. 2010; 70:2406-2414. [PubMed: 20215514]

19. Daniel CR, Cross AJ, Graubard BI, et al. Prospective investigation of poultry and fish intake in relation to cancer risk. Cancer Prev Res (Phila). 2011; 4:1903-1911. [PubMed: 21803982]

20. Choi Y, Song S, Song Y, et al. Consumption of red and processed meat and esophageal cancer risk: meta-analysis. World J Gastroenterol. 2013; 19:1020-1029. [PubMed: 23467465]

21. Kubo A, Corley DA, Jensen CD, et al. Dietary factors and the risks of oesophageal adenocarcinoma and Barrett's oesophagus. Nutr Res Rev. 2010; 23:230-246. [PubMed: 20624335] 
22. O'Doherty MG, Cantwell MM, Murray LJ, et al. Dietary fat and meat intakes and risk of reflux esophagitis, Barrett's esophagus and esophageal adenocarcinoma. Int J Cancer. 2011; 129:14931502. [PubMed: 21455992]

23. Song $\mathrm{P}, \mathrm{Lu} \mathrm{M}$, Yin Q, et al. Red meat consumption and stomach cancer risk: a meta-analysis. J Cancer Res Clin Oncol. 2014; 140:979-992. [PubMed: 24682372]

24. Wu GD, Chen J, Hoffmann C, et al. Linking long-term dietary patterns with gut microbial enterotypes. Science. 2011; 334:105-108. [PubMed: 21885731]

25. Koeth RA, Wang Z, Levison BS, et al. Intestinal microbiota metabolism of L-carnitine, a nutrient in red meat, promotes atherosclerosis. Nat Med. 2013; 19:576-585. [PubMed: 23563705]

26. Wang Z, Klipfell E, Bennett BJ, et al. Gut flora metabolism of phosphatidylcholine promotes cardiovascular disease. Nature. 2011; 472:57-63. [PubMed: 21475195]

27. Watson WL. Cancer of the esophagus: some etiological considerations. Am J Roentgenology. 1939; 41:420-424.

28. Andrici J, Eslick GD. Mate consumption and the risk of esophageal squamous cell carcinoma: a meta-analysis. Dis Esophagus. 2013; 26:807-816. [PubMed: 22891687]

29. Islami F, Boffetta P, Ren JS, et al. High-temperature beverages and foods and esophageal cancer risk--a systematic review. Int J Cancer. 2009; 125:491-524. [PubMed: 19415743]

30. De Jong UW, Day NE, Mounier-Kuhn PL, et al. The relationship between the ingestion of hot coffee and intraoesophageal temperature. Gut. 1972; 13:24-30. [PubMed: 5060664]

31. Oze I, Matsuo K, Kawakita D, et al. Coffee and green tea consumption is associated with upper aerodigestive tract cancer in Japan. Int J Cancer. 2014; 135:391-400. [PubMed: 24310779]

32. Zamora-Ros R, Lujan-Barroso L, Bueno-de-Mesquita HB, et al. Tea and coffee consumption and risk of esophageal cancer: the European prospective investigation into cancer and nutrition study. Int J Cancer. 2014; 135:1470-1479. [PubMed: 24535727]

33. Islami F, Pourshams A, Nasrollahzadeh D, et al. Tea drinking habits and oesophageal cancer in a high risk area in northern Iran: population based case-control study. BMJ. 2009; 338:b929. [PubMed: 19325180]

34. Dar NA, Bhat GA, Shah IA, et al. Salt tea consumption and esophageal cancer, a possible role of alkaline beverages in esophageal carcinogenesis. Int J Cancer. 2014; 10

35. Nechuta S, Shu XO, Li HL, et al. Prospective cohort study of tea consumption and risk of digestive system cancers: results from the Shanghai Women's Health Study. Am J Clin Nutr. 2012; 96:1056-1063. [PubMed: 23053557]

36. Sang LX, Chang B, Li XH, et al. Green tea consumption and risk of esophageal cancer: a metaanalysis of published epidemiological studies. Nutr Cancer. 2013; 65:802-812. [PubMed: 23909723]

37. Tewes FJ, Koo LC, Meisgen TJ, et al. Lung cancer risk and mutagenicity of tea. Environ Res. 1990; 52:23-33. [PubMed: 2351126]

38. Dorado G, Barbancho M, Pueyo C. Coffee is highly mutagenic in the L-arabinose resistance test in Salmonella typhimurium. Environ Mutagen. 1987; 9:251-260. [PubMed: 3552646]

39. Lambert JD, Yang CS. Mechanisms of cancer prevention by tea constituents. J Nutr. 2003; 133:3262S-3267S. [PubMed: 14519824]

40. Yang CS, Lambert JD, Ju J, et al. Tea and cancer prevention: molecular mechanisms and human relevance. Toxicol Appl Pharmacol. 2007; 224:265-273. [PubMed: 17234229]

41. Kamangar F, Strickland PT, Pourshams A, et al. High exposure to polycyclic aromatic hydrocarbons may contribute to high risk of esophageal cancer in northeastern Iran. Anticancer Res. 2005; 25:425-428. [PubMed: 15816606]

42. Yang CS, Wang H, Li GX, et al. Cancer prevention by tea: Evidence from laboratory studies. Pharmacol Res. 2011; 64:113-122. [PubMed: 21397027]

43. Pae M, Wu D. Immunomodulating effects of epigallocatechin-3-gallate from green tea: mechanisms and applications. Food Funct. 2013; 4:1287-1303. [PubMed: 23835657]

44. Sang LX, Chang B, Li XH, et al. Green tea consumption and risk of esophageal cancer: a metaanalysis of published epidemiological studies. Nutr Cancer. 2013; 65:802-812. [PubMed: 23909723] 
45. Pourshams A, Khademi H, Malekshah AF, et al. Cohort Profile: The Golestan Cohort Study--a prospective study of oesophageal cancer in northern Iran. Int J Epidemiol. 2010; 39:52-59. [PubMed: 19332502]

46. Myung SK, Bae WK, Oh SM, et al. Green tea consumption and risk of stomach cancer: a metaanalysis of epidemiologic studies. Int J Cancer. 2009; 124:670-677. [PubMed: 18973231]

47. Sun CL, Yuan JM, Koh WP, et al. Green tea, black tea and colorectal cancer risk: a meta-analysis of epidemiologic studies. Carcinogenesis. 2006; 27:1301-1309. [PubMed: 16638787]

48. Lampe JW. Health effects of vegetables and fruit: assessing mechanisms of action in human experimental studies. Am J Clin Nutr. 1999; 70:475S-490S. [PubMed: 10479220]

49. Stanner SA, Hughes J, Kelly CN, et al. A review of the epidemiological evidence for the 'antioxidant hypothesis'. Public Health Nutr. 2004; 7:407-422. [PubMed: 15153272]

50. Bailey RL, Gahche JJ, Miller PE, et al. Why US adults use dietary supplements. JAMA Intern Med. 2013; 173:355-361. [PubMed: 23381623] 


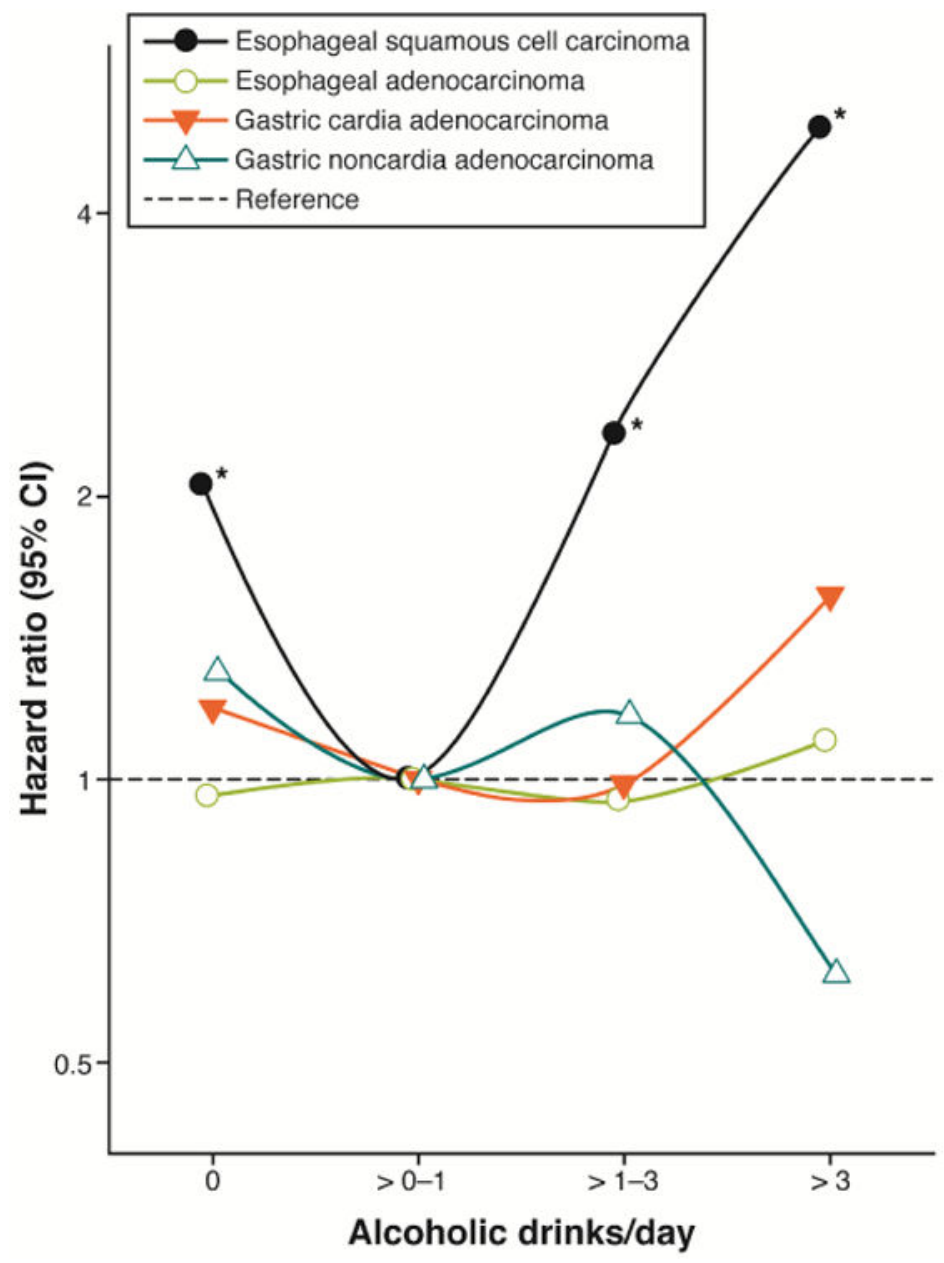

Figure 1. Associations between categories of alcoholic beverage intake and risk of upper GI Cancers

Note: Data from the NIH-AARP Diet and Health Study cohort. Point estimates marked with an asterisk are significantly different from unity. Adapted from Freedman et al. American Journal of Epidemiology 2007. 


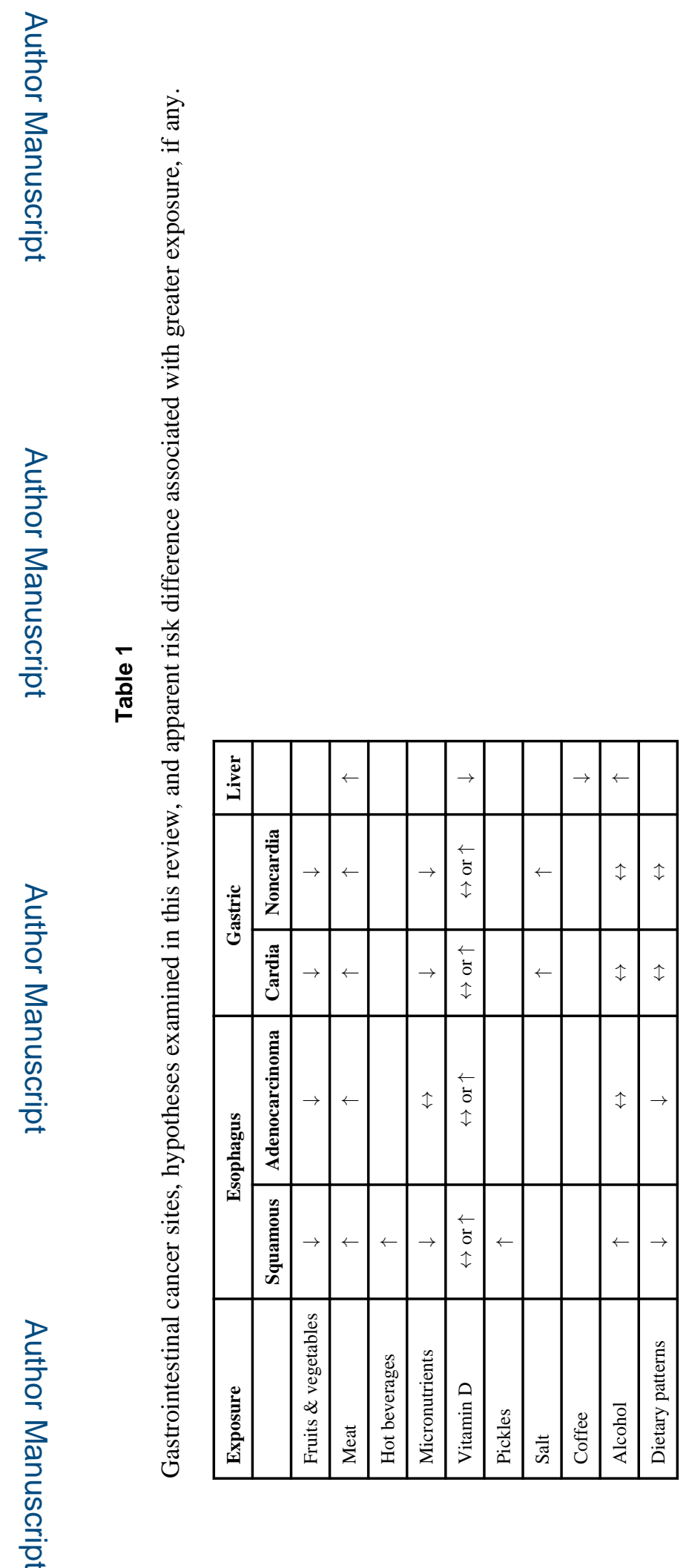

Gastroenterology. Author manuscript; available in PMC 2016 May 01. 